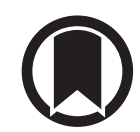

CrossMark

\title{
KRAS oncogene in lung cancer: focus on molecularly driven clinical trials
}

\author{
Emmanuelle Kempf $f^{1,2}$, Benoît Rousseau ${ }^{2,3}$, Benjamin Besse $e^{4,5}$ \\ and Luis Paz-Ares ${ }^{1}$
}

Affiliations: ${ }^{1}$ Dept of Medical Oncology, Virgen del Rocio Teaching Hospital, Instituto de Biomedicina de Sevilla - IBIS, Seville, Spain. ${ }^{2}$ Dept of Medical Oncology, Pharmacology Unit, AP-HP, Henri Mondor Teaching Hospital, Créteil, France. ${ }^{3}$ Université Paris-Est, VIC DHU, Inserm U 955, Team 18, UPEC, Créteil, France. ${ }^{4}$ Dept of Cancer Medicine, Gustave Roussy Cancer Campus, Villejuif, France. ${ }^{5}$ Paris-Sud University, Inserm U981, Paris, France.

Correspondence: Emmanuelle Kempf, Pharmacology Unit, Henri Mondor University Hospital, 51 Avenue du Maréchal de Lattre de Tassigny 94010 Créteil Cedex, France. E-mail: emmalakempf.pro

ABSTRACT KRAS mutations are the most frequent molecular abnormalities found in one out of four nonsmall cell lung cancers (NSCLC). Their incidence increases in cases of adenocarcinoma, smokers and Caucasian patients. Their negative value in terms of prognosis and responsiveness to both standard chemotherapy and targeted therapies remains under debate. Many drugs have been developed specifically for KRAS-mutated NSCLC patients. Direct inhibition of RAS activation failed to show any clinical efficacy. Inhibition of downstream targets of the mitogen-activated protein kinase (MEK) pathway is a promising strategy: phase II combinations of MEK 1/2 kinase inhibitors with chemotherapy doubled patients' clinical outcomes. One phase III trial in such a setting is ongoing. Double inhibition of MEK and epidermal growth factor receptor proteins is currently being assessed in early-phase trials. The association with mammalian target of rapamycin pathway inhibition leads to non-manageable toxicity. Other strategies, such as inhibition of molecular heat-shock proteins 90 or focal adhesion kinase are currently assessed. Abemaciclib, a cyclindependent kinase 4/6 inhibitor, showed promising results in a phase I trial, with a $54 \%$ disease control rate. Results of an ongoing phase III trial are warranted. Immunotherapy might be the next relevant step in KRAS-mutated NSCLC management due to the high burden of associated mutations and neo-antigens.

@ERSpublications

MEK inhibition and immunotherapy are very promising therapeutic advances in $K R A S$-mutated nonsmall cell lung cancer http://ow.ly/U2ohp

\section{KRAS mutations in lung cancer: epidemiology and clinical outcomes}

Since the beginning of the 21st century, the paradigm of precision medicine has shaken up the landscape of lung cancer classification and treatment. The discovery of cancer-related driver molecular abnormalities led to the development of efficient targeted therapies. RAS proteins are GTP kinases, discovered in the 1960s, whose GTP-RAS active isoform stimulates several pathways involved in cellular growth. V-Ki-ras2 Kirsten rat sarcoma viral oncogene homologue (KRAS) mutations are found in $\sim 25-35 \%$ of newly diagnosed nonsmall cell lung cancer (NSCLC), with a higher proportion in the adenocarcinoma subtype $[1,2]$. Figure 1 summarises the main amino acid substitutions and genomic features that are associated with KRAS mutations in NSCLC [3, 4]. Other molecular abnormalities related to RAS pathway activation are diagnosed in $25 \%$ of NSCLC cases, such as epidermal growth factor receptor (EGFR) (10-23\%), BRAF mutations (2\%), MET amplifications (2\%), human epidermal growth factor (HER)2 (1\%) and NRAS $(0.2 \%)$ mutations. Loss of the negative regulator neurofibromin is found in $\sim 11 \%$ of other cases.

Received: Sept 162015 | Accepted after revision: Oct 122015

Conflict of interest: None declared.

Provenance: Submitted article, peer reviewed.

Copyright OERS 2016. ERR articles are open access and distributed under the terms of the Creative Commons Attribution Non-Commercial Licence 4.0. 
FIGURE 1 Main genomic features of $\mathrm{V}$-Ki-ras2 Kirsten rat sarcoma viral oncogene homologue (KRAS)mutated nonsmall cell lung cancer (NSCLC). EGFR: epidermal growth factor receptor.

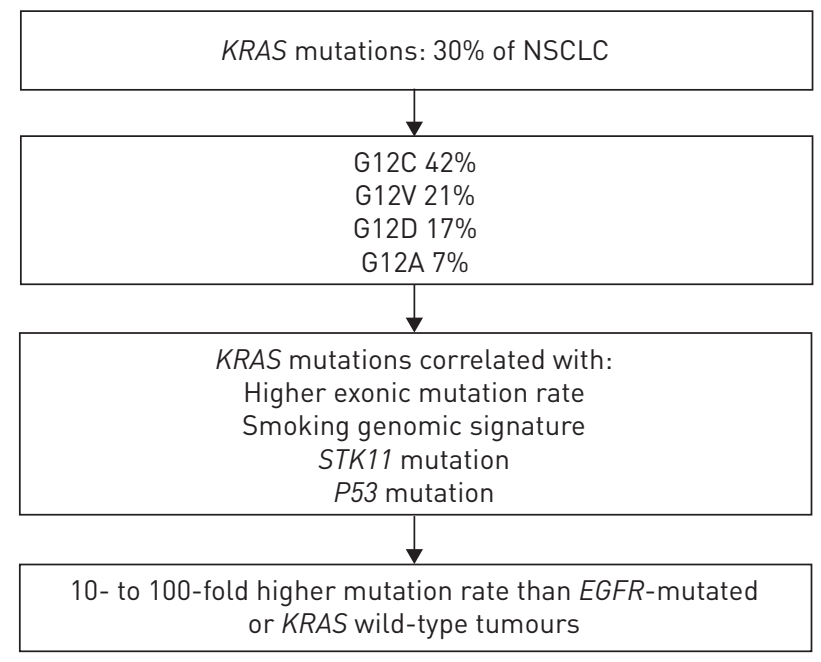

Among KRAS mutations, G12C, G12V, G12D, G12A and other G12 and G13 mutations are diagnosed in approximately $40 \%, 21 \%, 17 \%, 10 \%$ and $12 \%$ of cases, respectively [3]. Patients' smoking history underlies the molecular profile of NSCLC, from the point of view of quantitative and qualitative molecular alterations. Incidence of KRAS mutations reaches $25-35 \%$ in smokers and only $5 \%$ in nonsmokers $[5,6]$. Type of KRAS mutation is related to prior smoking history. In never-smokers, the most common KRAS mutation is G12D (56\%), and G12C is the most frequent mutation among former and current smokers (41\%). Never-smokers are more likely than former and current smokers to have $G>A$ transition mutations, whereas $G>T$ transversion mutations are the most common nucleotide change in former and current smokers [3, 7]. It is an early oncogenic event: patients harbouring such mutations have a significantly longer time since quitting smoking ( 9 versus 3 years, $\mathrm{p}=0.039$ ), with no added difference regarding other tobacco consumption characteristics [8]. KRAS mutations are ethnicity driven, since they are found in only $10 \%$ of Asian patients [6]. They seem to be associated with poorer prognosis in NSCLC. In two meta-analyses, KRAS mutations led to a $30 \%$ relative mortality over-risk $[9,10]$. Nevertheless, EGFR mutation status was not taken into account, which may have led to an overestimation of control arm outcomes: KRAS and EGFR mutations are exclusive from each other, and EGFR-mutated NSCLC has the better prognosis. A recent review confirmed the negative prognostic value of KRAS mutations. In this study, 265 KRAS-mutant NSCLC patients experienced shorter median overall survival compared to KRAS wild-type NSCLC, after excluding EGFR-mutant cases (43 versus 55 months, $\mathrm{p}<0.0001)$ [11]. Tumour stage might interfere with the prognostic interpretation of KRAS mutations [12]. Types of mutated codons seem to have a prognostic value [13]. Analysis was performed on $300 \mathrm{KRAS}$-mutant NSCLC from four randomised clinical trials in the postoperative setting by the Lung Adjuvant Cisplatin Evaluation (LACE)-BIO collaborative group. KRAS-mutant cases allocated to the observation arm were more likely to experience a second primary tumour occurrence (hazard ratio (HR) 2.76, 95\% CI 1.34-5.70; $\mathrm{p}=0.005$ ) [14]. In two studies, G12V codon 12 transversions were associated with worse survival [11, 15].

Some retrospective data suggest that KRAS mutations might present a negative predictive role of responsiveness to chemotherapy [16], especially when exploring types of KRAS molecular abnormalities. In the LACE-BIO collaborative group study, patients harbouring codon 13 mutations experienced much worse outcomes when allocated to the chemotherapy group compared to the observation arm (HR 5.78, 95\% CI 2.06-16.22; $\mathrm{p}<0.001$, interaction=0.002), suggesting a deleterious effect of adjuvant chemotherapy in this subgroup of patients [14]. In KRAS-mutant NSCLC, type of chemotherapy regimen seems to lead to similar clinical outcomes [17, 18]. Until 2010, KRAS mutations were considered negative predictive factors in advanced NSCLC patients treated by inhibitors of the tyrosine kinase domain (TKI) of the EGFR, according to the results of two meta-analyses evaluating both erlotinib and gefitinib $[5,19,20]$. When focusing exclusively on EGFR wild-type cases, KRAS mutations might not keep this negative predictive value $[18,21-23]$. To the best of our knowledge, data related to clinical benefits of bevacizumab according to KRAS mutational status are lacking.

\section{Molecularly driven trials: results and further directions in KRAS-mutated NSCLC}

All the results of patients' clinical outcomes described are related to KRAS-mutated cases.

\section{Pathways involved in RAS activation}

The impaired GTPase activity related to KRAS mutations leads to higher cytoplasmic concentrations of prooncogenic GTP-KRAS compound. To directly inhibit RAS protein activity, three strategies were used (table 1). 
TABLE 1 Clinical outcomes related to evaluated drugs in KRAS-mutated nonsmall cell lung cancer patients

\begin{tabular}{|c|c|c|c|c|c|c|}
\hline Trial arm [ref.] & Patients & Previous lines & ORR \% & DCR \% & Median PFS months & Median OS months \\
\hline \multicolumn{7}{|l|}{ MAPK pathway } \\
\hline Salirasib [24] & 33 & Any & 0 & 33.3 & & \\
\hline Sorafenib [26] & 59 & $\geqslant 1$ & 8.5 & 50.8 & 2.3 & 5.3 \\
\hline Sorafenib & 34 & $\geqslant 2$ & 2.9 & 44.1 & 2.6 & 6.4 \\
\hline versus placebo [27] & 34 & $\geqslant 2$ & 0 & 7.6 & $\begin{array}{c}1.7 \text { (HR 0.46, } \\
95 \% \mathrm{Cl} 0.25-0.82 \\
p=0.007 \text { ) }\end{array}$ & $\begin{array}{c}5.1 \text { (HR 0.76, } \\
95 \% \mathrm{Cl} 0.45-1.26 \\
p=0.279)\end{array}$ \\
\hline Sorafenib & 14 & $\geqslant 1$ & & 79 & & \\
\hline versus erlotinib & 7 & $\geqslant 1$ & & 14 & & \\
\hline versus erlotinib + bexarotene & 3 & $\geqslant 1$ & & 33 & & \\
\hline versus vandetanib [28] & 14 & $\geqslant 1$ & & 0 & & \\
\hline Selumetinib & 9 & $\geqslant 1$ & 0 & & 3.9 & \\
\hline versus selumetinib + erlotinib [29] & 30 & $\geqslant 1$ & 6.7 & & 4.5 & \\
\hline versus docetaxel [31] & 43 & 1 & 11.6 & 74.4 & $\begin{array}{c}2.8 \text { (HR 1.23, } \\
95 \% \mathrm{Cl} 0.81-1.87 ; \\
p=0.316 \text { ) }\end{array}$ & $\begin{array}{l}\text { Unreached (HR 0.97, } \\
95 \% \mathrm{Cl} 0.52-1.83 \\
\text { p=0.934) }\end{array}$ \\
\hline Trametinib + docetaxel [32] & 22 & $\geqslant 1$ & 13.6 & 61 & & \\
\hline Trametinib + pemetrexed [33] & 20 & $\geqslant 1$ & 75 & 65 & & \\
\hline \multicolumn{7}{|l|}{ mTOR inhibitors } \\
\hline Ridaforolimus [34] & 79 & $\geqslant 1$ & & 35.4 & & \\
\hline Ridaforolimus & 14 & $\begin{array}{c}\geqslant 1 \text { SD } \\
\text { after } 8 \text { weeks ridaforolimus }\end{array}$ & & & 4 & 18 \\
\hline versus placebo [34] & 14 & $\begin{array}{c}\geqslant 1 \text { SD } \\
\text { after } 8 \text { weeks ridaforolimus }\end{array}$ & & & 2 (HR 0.36, $p=0.013$ ) & 5 (HR 0.46, p=0.09) \\
\hline \multicolumn{7}{|l|}{ Hsp90 inhibitor } \\
\hline
\end{tabular}

First, targeting the nucleotide binding to RAS with a competitive inhibitor to prevent GTP-KRAS formation [36-39]. Second, improving GTPase activity of KRAS-mutated cells. High cytoplasmic guanine concentrations, very high affinity of the nucleotide and unknown KRAS binding sites hinder the clinical development of these options. Finally, RAS activation may be inhibited by targeting its endomembrane binding through phosphodiesterase- $\delta$ [40]. Yet a phase II trial testing salirasib, an inhibitor of such binding, failed to show any clinical benefit [24].

If direct blocking of RAS has not yet demonstrated its efficacy, inhibition of downstream targets of the mitogen-activated protein kinase (MAPK) cascade may be a more promising strategy. Sorafenib is an oral multitarget TKI which inhibits RAF and related transmembrane receptors. Phase II trials showed promising results with a disease control rate of $\sim 50 \%[25,26,28]$. Yet subgroup analyses performed in the KRAS-mutated group of the phase III MISSION trial did not reveal any specific efficacy of sorafenib in the third or fourth chemotherapy line [27].

Selumetinib is a non-ATP competitive oral inhibitor of mitogen-activated protein kinase kinase (MEK)1/2, which was combined with docetaxel in a second-line phase II trial. Compared to docetaxel monotherapy, progression-free survival and overall survival were doubled in the experimental arm with a good tolerance profile [30]. Yet the outcome in the control arm was poorer than expected, arm populations were unbalanced regarding prognostic factors and data regarding efficacy of the experimental combination in KRAS wild-type NSCLC patients are lacking. The SELECT-1 phase III trial is ongoing in this indication (clinicaltrials.gov: NCT01933932) (table 2). Trametinib belongs to the same molecular class as selumetinib and has been developed in BRAF-mutated metastatic melanoma. A second-line phase II trial comparing trametinib to docetaxel revealed similar survival outcomes, while grade 4 toxicity occurred only in the experimental arm [31]. Two phase I/Ib trials evaluating trametinib combinations with docetaxel 


\begin{tabular}{|c|c|c|c|}
\hline \multicolumn{4}{|l|}{ MEK inhibitors } \\
\hline Selumetinib + docetaxel (versus docetaxel) & NCT01933932 & III & NSCLC \\
\hline Trametinib + chemoradiation & NCT01912625 & 1 & Unresectable NSCLC \\
\hline PD-0325901 + palbociclib & NCT02022982 & I/II & NSCLC and other solid tumours \\
\hline MEK162 + BYL719 & NCT01449058 & $\mathrm{lb}$ & All solid tumours \\
\hline MEK162 + erlotinib & NCT01859026 & 1 & NSCLC \\
\hline PD-0325901 + dacomitinib & NCT02039336 & I & NSCLC \\
\hline \multicolumn{4}{|l|}{ Other } \\
\hline BIND-014 & NCT02283320 & II & NSCLC \\
\hline Bortezomib & NCT01833143 & II & NSCLC \\
\hline Retaspimycin $\mathrm{HCl}(\mathrm{IPI}-504)+$ everolimus & NCT01427946 & $\mathrm{Ib} / \mathrm{II}$ & NSCLC \\
\hline
\end{tabular}

and pemetrexed, respectively, as a second line showed a disease control rate of $\sim 60 \%[32,33]$. The radiosensitising effect of trametinib is currently being assessed in combination with a carboplatinpaclitaxel regimen (clinicaltrials.gov: NCT01912625). MEK162 and PD-0325901 are second-generation MEK1/2 inhibitors currently evaluated in molecularly driven phase I/II trials (clinicaltrials.gov: NCT02022982). Based on a genomic classification, the double inhibition of MEK and EGFR proteins is a promising therapeutic strategy [41]. Early-phase trials are assessing currently such associations: MEK162erlotinib (clinicaltrials.gov: NCT01859026) or PD-0325901-dacomitinib (clinicaltrials.gov: NCT02039336).

The phosphatidylinositol-3-kinase (PI3K)-Akt-mammalian target of rapamycin (mTOR) pathway is a parallel signal transduction pathway. The single inhibition of mTOR by ridaforalimus led to disappointing results in KRAS-mutated NSCLC [34]. NVP-BEZ235, a dual PI3K-mTOR inhibitor, failed to demonstrate promising preclinical results in this context. PI3K-Akt-mTOR pathway inhibition may circumvent resistance to MEK inhibition in KRAS-mutated NSCLC. Yet the combination of both PI3K-Akt-mTOR and RAS-MEK-ERK inhibitors leads to nonmanageable toxicity [42, 43].

\section{Other strategies}

Bortezomib (Velcade) is a proteasome inhibitor which is currently evaluated in an ongoing molecularly driven phase II trial (NCT01833143). Molecular heat shock proteins (Hsp)90 are chaperones that assist proteins to fold properly, stabilising them against cellular stress and preventing their proteasomal degradation. Ganetespib and retaspimycin $\mathrm{HCl}$ (IPI-504) are Hsp90 inhibitors. A phase II study assessing ganetespib showed similar clinical outcomes according to the KRAS mutational status of patients [35]. Results of a phase $\mathrm{Ib} / \mathrm{II}$ trial testing the association of retaspimycin with everolimus in KRAS-mutated advanced NSCLC patients are awaited (NCT01427946).

Focal adhesion kinase (FAK) is a nonreceptor tyrosine kinase involved in cellular matrix attachment, whose overexpression leads to tumoral growth. Defactinib (VS-6063) is a FAK inhibitor tested in a phase II trial among four cohorts of KRAS-mutated NSCLC patients (NCT01951690). The as-yet unpublished results were reported recently at the 16th World Conference on Lung Cancer and were negative.

Reovirus type 3 Dearing is a naturally occurring, nonpathogenic, double-stranded RNA virus isolated from $100 \%$ of the human respiratory and gastrointestinal tracts with some antitumoral activity among RAS pathway activation tumours. It has been tested in a phase II trial in association with a carboplatin/ paclitaxel regimen in RAS pathway-activated NSCLC patients (NCT01951690).

BIND-014 is a drug made of docetaxel nanoparticles and is currently evaluated in a phase II trial among KRAS-mutated or squamous-cell NSCLC patients, as a second-line therapy (NCT02283320).

Abemaciclib (LY2835219) is a cell cycle inhibitor selective for the cyclin-dependent kinases CDK4 and CDK6. Promising results of a phase I trial were presented at the American Society of Clinical Oncology congress in 2014: in a relapse setting, disease control rate reached 54\% in KRAS-mutated NSCLC and toxicity was manageable. The JUNIPER phase III clinical trial is currently ongoing (NCT02152631). KRAS-mutated NSCLC patients are randomised into the monotherapy experimental arm or into the erlotinib control arm, after failure of a platinum-based chemotherapy line. 


\section{Discussion: is immunotherapy the next step in the treatment of KRAS-mutated NSCLC patients?}

Treating KRAS-mutated NSCLC patients selectively remains a challenge in the era of precision medicine. Several reasons are involved: 1 ) the absence of oncogenic addiction leading to cancer primary resistance to MAPK pathway targeted therapies; 2) distinct patterns of KRAS-mutated NSCLC; 3) other forms of RAS pathway activation such as RAS overexpression or loss of RAS negative regulator (neurofibromin); 4) heterogeneity between the primary tumour site and metastases [44]; and 5) distinct methods of mutation assessment between solid and liquid biopsies [45]. MEK1/2 inhibitors are promising drugs in this setting. Yet, immunotherapy might be a relevant translational research option [46] since: 1) KRAS-mutated NSCLCs occur more frequently among smokers and tobacco-induced tumours present a higher burden of mutations and neo-antigens; 2) KRAS mutations are associated with a high number of mutations [47]; and 3) among responders to programmed cell death (PD)-1 inhibitor, 50\% of cases harbour a KRAS mutation, while this concerns only one out of 17 nonreponders [48].

\section{References}

1 Kris MG, Johnson BE, Kwiatkowski DJ, et al. Identification of driver mutations in tumor specimens from 1,000 patients with lung adenocarcinoma: the NCI's Lung Cancer Mutation Consortium (LCMC). J Clin Oncol 2011; 29: Suppl., CRA7506.

2 Barlesi F, Blons H, Beau-Faller M, et al. Biomarkers (BM) France: results of routine EGFR, HER2, KRAS, BRAF, PI3KCA mutations detection and EML4-ALK gene fusion assessment on the first 10,000 non-small cell lung cancer (NSCLC) patients (pts). J Clin Oncol 2013; 31: Suppl., 8000.

3 Dogan S, Shen R, Ang DC, et al. Molecular epidemiology of EGFR and KRAS mutations in 3,026 lung adenocarcinomas: higher susceptibility of women to smoking-related KRAS-mutant cancers. Clin Cancer Res 2012; 18: 6169-6177.

4 Imielinski M, Berger AH, Hammerman PS, et al. Mapping the hallmarks of lung adenocarcinoma with massively parallel sequencing. Cell 2012; 150: 1107-1120.

5 Mao C, Qiu LX, Liao RY, et al. KRAS mutations and resistance to EGFR-TKIs treatment in patients with non-small cell lung cancer: a meta-analysis of 22 studies. Lung Cancer 2010; 69: 272-278.

6 Dearden S, Stevens J, Wu YL, et al. Mutation incidence and coincidence in non small-cell lung cancer: meta-analyses by ethnicity and histology (mutMap). Ann Oncol 2013; 24: 2371-2376.

7 Riely GJ, Kris MG, Rosenbaum D, et al. Frequency and distinctive spectrum of KRAS mutations in never smokers with lung adenocarcinoma. Clin Cancer Res 2008; 14: 5731-5734.

8 Thunnissen FB, Prinsen C, Hol B, et al. Smoking history and lung carcinoma: KRAS mutation is an early hit in lung adenocarcinoma development. Lung Cancer 2012; 75: 156-160.

9 Mascaux C, Iannino N, Martin B, et al. The role of RAS oncogene in survival of patients with lung cancer: a systematic review of the literature with meta-analysis. Br J Cancer 2005; 92: 131-139.

10 Meng D, Yuan M, Li X, et al. Prognostic value of K-RAS mutations in patients with non-small cell lung cancer: a systematic review with meta-analysis. Lung Cancer 2013; 81: 1-10.

11 Renaud S, Falcoz PE, Schaeffer M, et al. Prognostic value of the KRAS G12V mutation in 841 surgically resected Caucasian lung adenocarcinoma cases. Br J Cancer 2015; 113: 1206-1215.

12 Yu HA, Sima CS, Hellmann MD, et al. Differences in the survival of patients with recurrent versus de novo metastatic KRAS-mutant and EGFR-mutant lung adenocarcinomas. Cancer 2015; 121: 2078-2082.

13 Villaruz LC, Socinski MA, Cunningham DE, et al. The prognostic and predictive value of KRAS oncogene substitutions in lung adenocarcinoma. Cancer 2013; 119: 2268-2274.

14 Shepherd FA, Domerg C, Hainaut P, et al. Pooled analysis of the prognostic and predictive effects of KRAS mutation status and KRAS mutation subtype in early-stage resected non-small-cell lung cancer in four trials of adjuvant chemotherapy. J Clin Oncol 2013; 31: 2173-2181.

15 Ihle NT, Byers LA, Kim ES, et al. Effect of KRAS oncogene substitutions on protein behavior: implications for signaling and clinical outcome. J Natl Cancer Inst 2012; 104: 228-239.

16 Macerelli M, Caramella C, Faivre L, et al. Does KRAS mutational status predict chemoresistance in advanced non-small cell lung cancer (NSCLC)? Lung Cancer 2014; 83: 383-388.

17 Dong X, Zhao X, Hao Y, et al. Response to first-line chemotherapy in patients with non-small-cell lung cancer according to epidermal growth factor receptor and K-RAS mutation status. Clin Lung Cancer 2013; 14: 680-687.

18 Sun JM, Hwang DW, Ahn JS, et al. Prognostic and predictive value of KRAS mutations in advanced non-small cell lung cancer. PLoS One 2013; 8: e64816.

19 Ludovini V, Bianconi F, Pistola L, et al. Phosphoinositide-3-kinase catalytic alpha and KRAS mutations are important predictors of resistance to therapy with epidermal growth factor receptor tyrosine kinase inhibitors in patients with advanced non-small cell lung cancer. J Thorac Oncol 2011; 6: 707-715.

20 Linardou H, Dahabreh IJ, Kanaloupiti D, et al. Assessment of somatic $k$-RAS mutations as a mechanism associated with resistance to EGFR-targeted agents: a systematic review and meta-analysis of studies in advanced non-small-cell lung cancer and metastatic colorectal cáncer. Lancet Oncol 2008; 9: 962-972.

21 Garassino MC, Martelli O, Broggini M, et al. Erlotinib versus docetaxel as second-line treatment of patients with advanced non-small-cell lung cancer and wild-type EGFR tumours (TAILOR): a randomised controlled trial. Lancet Oncol 2013; 14: 981-988.

22 Metro G, Chiari R, Duranti S, et al. Impact of specific mutant KRAS on clinical outcome of EGFR-TKI-treated advanced non-small cell lung cancer patients with an EGFR wild type genotype. Lung Cancer 2012; 78: 81-86.

23 Oxnard GR, Jänne PA. KRAS wild-type lung cancer: a moving target in an era of genotype migration. J Clin Oncol 2012; 30: 3322-3324.

24 Riely GJ, Johnson ML, Medina C, et al. A phase II trial of salirasib in patients with lung adenocarcinomas with KRAS mutations. J Thorac Oncol 2011; 6: 1435-1437. 


\section{AKT/mTOR and RAS/MEK/ERK pathways in patients with advanced cancer. Clin Cancer Res 2012; 18:} 2316-2325.

43 Tolcher AW, Patnaik A, Papadopoulos KP, et al. Phase I study of the MEK inhibitor trametinib in combination with the AKT inhibitor afuresertib in patients with solid tumors and multiple myeloma. Cancer Chemother Pharmacol 2015; 75: 183-189.

44 Kalikaki A, Koutsopoulos A, Trypaki M, et al. Comparison of EGFR and K-RAS gene status between primary tumours and corresponding metastases in NSCLC. Br J Cancer 2008; 99: 923-929.

45 Nygaard AD, Garm Spindler KL, Pallisgaard N, et al. The prognostic value of KRAS mutated plasma DNA in advanced non-small cell lung cancer. Lung Cancer 2013; 79: 312-317.

46 Gjertsen MK, Gaudernack G. Mutated Ras peptides as vaccines in immunotherapy of cancer. Vox Sang 1998; 74: Suppl. 2, 489-495.

47 Imielinski M, Berger AH, Hammerman PS, et al. Mapping the hallmarks of lung adenocarcinoma with massively parallel sequencing. Cell 2012; 150: 1107-1120.

48 Rizvi NA, Hellmann MD, Snyder A, et al. Mutational landscape determines sensitivity to PD-1 blockade in non-small cell lung cancer. Science 2015; 348: 124-128. 